\title{
Demonstration of Displacement- and Frequency-Noise-Free Laser Interferometry Using Bidirectional Mach-Zehnder Interferometers
}

\author{
Shuichi Sato, ${ }^{1, *}$ Keiko Kokeyama, ${ }^{2}$ Robert L. Ward, ${ }^{3}$ Seiji Kawamura, ${ }^{1}$ Yanbei Chen, ${ }^{4}$ Archana Pai, ${ }^{4}$ and Kentaro Somiya ${ }^{4}$ \\ ${ }^{1}$ TAMA project, National Astronomical Observatory of Japan, 2-21-1, Mitaka, Osawa, Tokyo 181-8588 Japan \\ ${ }^{2}$ The Graduate School of Humanities and Sciences, Ochanomizu University, 2-1-1, Otsuka, Bunkyo-ku, Tokyo 112-8610, Japan \\ ${ }^{3}$ LIGO Project 18-34, California Institute of Technology, Pasadena, California 91125, USA \\ ${ }^{4}$ Max-Planck-Institut für Gravitationsphysik, Am Mühlenberg 1, 14476 Potsdam, Germany
}

(Received 27 August 2006; published 2 April 2007)

\begin{abstract}
We have demonstrated displacement- and frequency-noise-free laser interferometry (DFI) by partially implementing a recently proposed optical configuration using bidirectional Mach-Zehnder interferometers (MZIs). This partial implementation, the minimum necessary to be called DFI, has confirmed the essential feature of DFI: the combination of two MZI signals can be carried out in a way that cancels displacement noise of the mirrors while maintaining gravitational-wave signals. The attained maximum displacementnoise suppression was $45 \mathrm{~dB}$.
\end{abstract}

DOI: 10.1103/PhysRevLett.98.141101

PACS numbers: 04.80.Nn, 06.30.Ft, 95.55.Ym

Gravitational waves are ripples of space-time curvature. For an array of test masses separated from each other by a length scale $\sim L$, that is not too much bigger than the gravitational wavelength; the effect of gravitational waves can often be thought of as creating relative test-mass motions with order $\sim L h$, or creating additional time delays of order $L h / c$ for light pulses traveling between these test masses. One might then think that, in order to measure gravitational waves, we need to make sure that (i) our clocks must have timing errors lower than $\delta t \sim L h / c$, and that (ii) the test masses must follow geodesics within an accuracy of $\delta x \sim L h$. In fact, both are unnecessary. Point (i) was shown to be unnecessary more than a century ago, with the invention of interferometry - in which one light beam is split into two beams; each beam then measures the same quantity but with opposite sign, and then the two beams are subtracted coherently to eliminate the common fluctuations in light frequency while retaining the differential signal. An extended application of this idea in gravitational-wave detection is the so-called time-delay interferometry (TDI) [1]. Point (ii) is more specific to the problem of gravitational-wave detection, and was addressed only recently. As was shown theoretically in Refs. [2,3], when each test mass in an $N$-test-mass array sends and receives light pulses from all other test masses, and if the array contains enough test masses $[N>(d+2)$, where $d$ is the number of spatial dimensions of the array], then there exist combinations of time delays (i.e., signals) which do not sense test-mass motions or timing noises, but do (usually) sense gravitational waves. The existence of such noise-free signals relies on the fact that the gravitational-wave contribution to light pulse timing delays takes a form different from that of test-mass motions - we therefore can combine the pulse time delays in such a way as to eliminate the common test-mass motions, while retaining differential gravitational-wave signals. We will call configurations that cancel both timing and dis- placement noises displacement-noise-free interferometry (DFI).

Recently, practical optical designs of DFI using laser interferometry in two and three dimensions have been proposed [4]. In these configurations, the conventional, equal-arm Mach-Zehnder interferometer was used as a building block to eliminate laser noise. Four such MachZehnder interferometers were combined, in such a way that they form two pairs of counterpropagating Mach-Zehnder interferometers. Within each pair, the 2 Mach-Zehnders share the same beam splitters and folding mirrors; subtraction of their outputs balances out displacement noise from motions of the folding mirrors. The two pairs share the same beam splitters, which allows the elimination of beam splitter displacement noise. It should be mentioned that DFI configurations only work for nonzero frequencies, because at nearly zero frequency gravitational waves are indistinguishable from relative mirror motions. For configurations studied so far (without optical cavities), the transfer function from $h$ to effective phase shift is $(\omega L h / c)(\Omega L / c)^{\alpha}$ with $\omega$ the optical frequency, $\Omega$ the gravitational-wave frequency, and $\alpha=2$ for 3D configurations, and $\alpha=3$ for $2 \mathrm{D}$ configurations. This means we need to observe at frequencies near $c / L$ in order to have a transfer function comparable to conventional configurations. For instance, for the ground-based interferometers with arm lengths limited to several kilometers, $c / L$ becomes around $100 \mathrm{kHz}$, so it seems difficult for current DFI configurations to compete with them, which use optical cavities to increase the effective arm length targeting at gravitational waves at below $\mathrm{kHz}$ frequencies. Nevertheless, we speculate that it will eventually find realistic applications in future detectors, or at least become one factor to consider during the invention of new detector configurations. Another thing which should also be mentioned is that DFI can cancel out frequency and displacement noise of the component optics completely; however, 
any practical noises other than these, such as an intensity noise of the laser, noise arising from the scattered light, and electronic noise are potential noise sources in the DFI signal. Therefore, in practical application of DFI, these noises should be carefully suppressed to low enough levels.

In this Letter, we study DFI experimentally using a single pair of counterpropagating Mach-Zehnder interferometers, demonstrating the elimination of folding mirror displacement noise and the response to gravitational waves. We use lab-scale devices, with an $L \sim 1$ meter, which indicates a frequency scale of $\sim 100 \mathrm{MHz}$. Because of this high operation frequency, we simulate motions of folding mirrors with the phase shift induced by an EOM (electro-optical modulator) located at the position of the folding mirror. We place another EOM systematically at various other locations on the optical path, and use the phase shift it induces to map out the Green's function of the device's gravitational-wave response. We can do so because general relativity predicts that the effect of gravitational waves on our interferometers is equivalent to that of a medium with a time- and locationdependent refractive index, and because we do not intend to test general relativity in this experiment. In order to see this, we write the phase shift imposed by a gravitational wave (in the so-called transverse-traceless, or TT gauge) on a light beam emitted from $\mathbf{x}_{0}$ with spatial direction $\mathbf{N}$ :

$$
\phi^{\mathrm{GW}}(t)=\frac{\omega}{c} \int_{0}^{L} N_{i} N_{j} h_{i j}^{\mathrm{TT}}\left(t+l / c, \mathbf{x}_{0}+\mathbf{N} l\right) d l .
$$

Here $t$ is the emission time, $h_{i j}^{\mathrm{TT}}(t, \mathbf{x})$ is the gravitationalwave metric perturbation, see Eq. (6) of Ref. [4]. Except at places where the beams intersect (negligible), we have a unique $\mathbf{N}$ vector, up to a sign difference (because we have counterpropagating beams overlapped with each other), at all points the light might travel through. This leads to a truly unique $N_{i} N_{j}$ at each point, and the effect of the gravitational wave is the same as that of a medium with the following refractive-index distribution:

$$
n^{\mathrm{GW}}(t, \mathbf{x})=N_{i}(\mathbf{x}) N_{j}(\mathbf{x}) h_{i j}^{\mathrm{TT}}(t, \mathbf{x}) .
$$

As depicted in Fig. 1, the full 2D DFI configuration (essentially a squished version of the 3D configuration in Ref. [4]) is composed of two pairs of counterpropagating MZIs, for a total of four MZIs. Mach-Zehnder interferometers of equal-arm length operated on the midfringe are insensitive to laser frequency noise, and by superimposing two counterpropagating Mach-Zehnder interferometers on the same optical path, the displacements of the folding mirrors are sensed redundantly (once by each direction of the MZI) and can thus be unambiguously removed from the signal. An additional pair of counterpropagating MZIs can then be added, which share beam splitters with the first pair - this second pair allows redundant sensing of beam splitter displacements. As discussed in [4], the signals from the four MZIs can be combined in such a way as to cancel

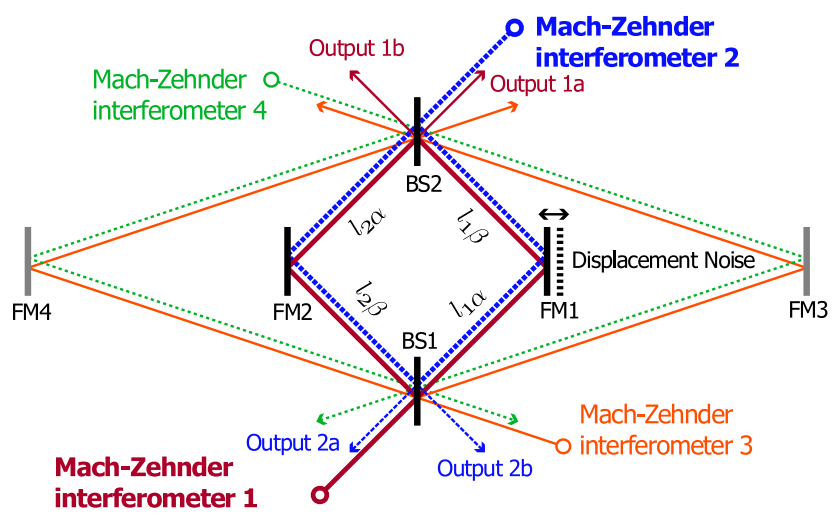

FIG. 1 (color online). Schematic of one possible 2D optical configuration of a displacement-noise-free interferometer. Light beams which follow common paths (thick, thin) travel in opposite directions, and the four MZIs share the two beam splitters (BS1 and BS2). The motion of each folding mirror (FM) is sensed by two MZIs, and the motion of the beam splitters is sensed by all four MZIs.

the displacements of all the optics while retaining sensitivity to gravitational radiation.

For this experiment we have constructed a partial-DFI composed of a single pair of counterpropagating MZIs to demonstrate the cancellation properties of the DFI. In Fig. 1, the inner pair of MZIs, composed of BS1, BS2, FM1, FM2, has equal-length arms with folding mirrors located at the midpoint of each arm. As the folding mirrors experience some displacement noise, the light field incident on those mirrors is phase modulated. Because the folding mirrors are at the midpoint of the arms, and there is light traveling in both directions in the MZI, these displacement-noise based phase modulations will arrive at output 1 and output 2, simultaneously. Direct subtraction of the outputs 1 and 2 then allows one to cancel the motion of the folding mirrors. Any signals which phase modulate the light and which do not arise at the midpoint will not arrive simultaneously at the outputs, and thus will not be fully cancelled.

The transfer function of the displacement motion of folding mirror (FM1) to the signal port is

$$
\begin{aligned}
& H_{D 1(a, b)}= \pm(\omega / c) \exp \left(-i l_{1 \beta} \Omega / c\right) \\
& H_{D 2(a, b)}= \pm(\omega / c) \exp \left(-i l_{1 \alpha} \Omega / c\right)
\end{aligned}
$$

where $c$ is the speed of light, $\omega$ is the laser frequency and $\Omega$ is a fourier frequency of the displacement motion. The signals from output ports $n a$ and $n b$ have opposite sign for phase variation, depending on the fringe condition where the MZIs are controlled. When the folding mirror is located at the exact center of the MZI arm (suppose $l_{1 \alpha}=l_{1 \beta}=l$ ), all four signals display an identical frequency dependence in their transfer function (with different sign) as 


$$
H_{D(1,2)(a, b)}= \pm(\omega / c) \exp (-i l \Omega / c),
$$

which enables the cancellation of signals due to displacement noise.

In contrast, the frequency response to the gravitationalwave $(\mathrm{GW})$ signal in a simplest case is given as

$$
H_{\mathrm{GW}(1,2)}= \pm(4 \omega / i \Omega) \exp (-i l \Omega / c) \sin ^{2}(l \Omega / 2 c)
$$

for symmetric MZIs $\left(l_{n \alpha}=l_{n \beta}=l\right.$ ), supposing normal incidence of GWs with most effective polarization. As a substitute for exact simulation of the GW signals, one can generate a phase modulation signal at a location that is not the midpoint of an arm, and thus map the response of the interferometer to nonsymmetrically located signals, by observing the difference in arrival times between the two detection ports.

The response function to such a signal is as given in Eq. (3). So, as a result, the signal combination of MZI 1 and MZI 2 is given as

$$
H_{\mathrm{sGW}}= \pm(\omega / c)\left(\exp \left(-i l_{1 \alpha} \Omega / c\right)-\exp \left(-i l_{1 \beta} \Omega / c\right)\right) \text {, }
$$

which shows complete cancellation of the displacement motion of the folding mirror, while allowing simulated$\mathrm{GW}$ signals to remain.

The practical experimental setup is shown in Fig. 2. The laser source is a commercial solid-state Nd:YAG laser (Lightwave Model-126) yielding $100 \mathrm{~mW}$ at $1064 \mathrm{~nm}$. The output beam is split into two, and each beam introduced to two MZIs after passing through Faraday isolators (FI), which allow detection of light exiting the counterpropagating MZI. The light paths of both MZIs are carefully adjusted and superposed on each other so that the two MZIs can exactly share the displacement noise and the effect of simulated-GW signals. The arm length of the MZIs affects the frequency where the MZI response to GW signal is maximized; in this experiment, the arm

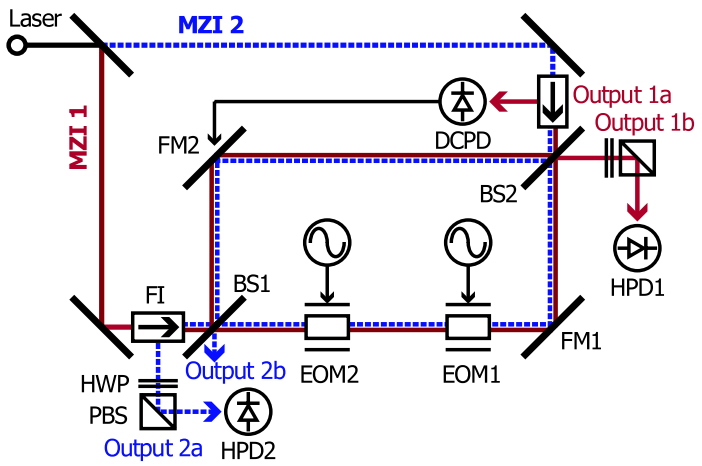

FIG. 2 (color online). The experimental setup of partial DFI. MZI 1 and MZI 2 share a common laser source and MachZehnder interferometer parts. The scale of the MZI was about $4 \mathrm{~m}$, for which purpose the optical paths of the MZI arms had to be folded several times on the optical table, which does not affect the DFI features in this experiment. lengths were chosen to be around $3.6 \mathrm{~m}$, to lower the peak frequency to several tens of $\mathrm{MHz}$. To enable high frequency operation, the phase modulation due to both displacement noise and a GW signal were simulated using electro-optic phase modulators located on one of the MZI arms. The displacement-noise simulator (EOM1) should be located at the exact center of the arm, so the position of EOM1 was carefully tuned, while the GW simulator (EOM2) was placed roughly $11 \mathrm{~cm}$ away from the BS1. The location of the EOM2 also determines the frequency of maximum sensitivity of DFI to the simulated-GW signal: a more asymmetrically placed EOM will give a lower peak frequency.

One of the output ports of MZI 1 (output 1a) was monitored with a de detector (DCPD) and an error signal created by subtracting a static offset; this signal was fed back to a PZT-actuated folding mirror (FM2) after appropriate filtering to give a midfringe locking control. The control bandwidth was very low, around $400 \mathrm{~Hz}$, so that the simulated signal by EOMs would not be suppressed by the fringe control feedback loop in the higher, more interesting frequency band. Once the fringe of the MZI 1 is controlled, that of MZI 2 is also automatically controlled because the two MZIs share common optical paths.

Other output lights (output $1 \mathrm{~b}$ and $2 \mathrm{a}$ ) were received with high-speed photodetectors (New Focus 1611-AC) to monitor differential optical path length variations. The polarization optics (HWP: half wave plate and PBS: polarizing beam splitter) just in front of both high-speed detec-

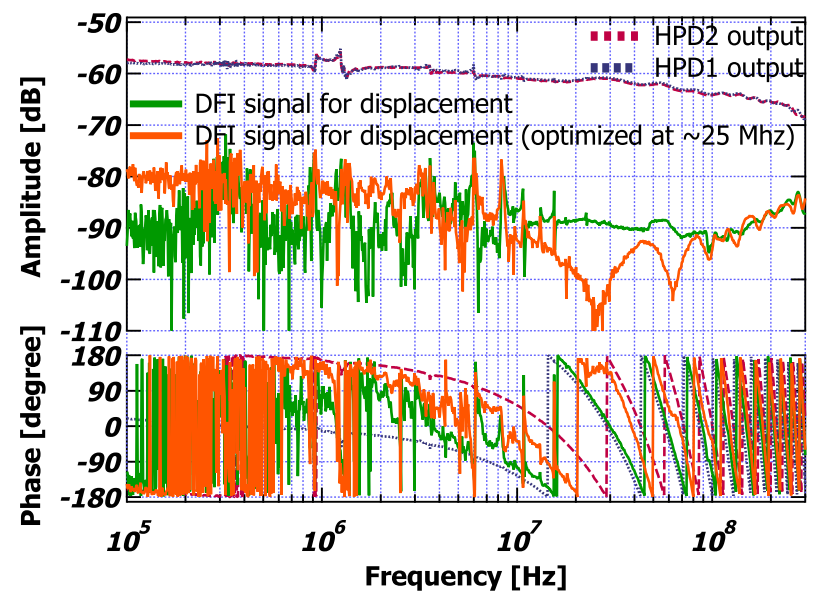

FIG. 3 (color online). The response functions from displacement noise to output signals. Dashed-red and dotted-blue curves are response to single detector HPD1 and HPD2, giving almost identical response in amplitude while opposite in sign. Therefore, summation of these two signals gives cancellation of displacement noise. The suppression depends on a delicate tuning of the balance between two signals. Approximately $30 \mathrm{~dB}$ of suppression can be seen in a wide frequency band from $10 \mathrm{kHz}$ to $300 \mathrm{MHz}$ in the green curve, meanwhile, the orange curve shows maximum suppression exceeding $45 \mathrm{~dB}$ at around $25 \mathrm{MHz}$. 


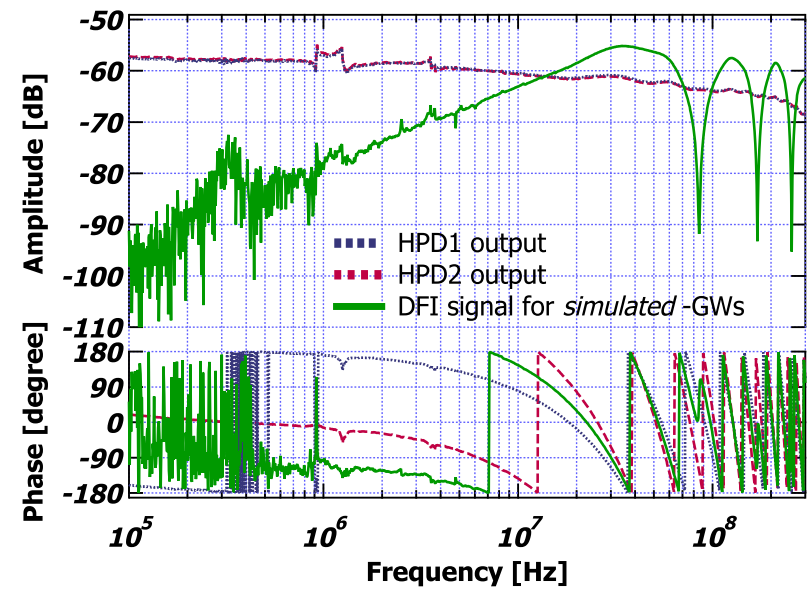

FIG. 4 (color online). The response functions from simulatedGW inputs to output signals. HPD1 and HPD2 response show almost the same response again in amplitude, whereas the phase delays in the higher frequency band are essentially different from that of displacement noise, which is the very reason why the summed signal (DFI signal) remains GW sensitive as is shown in the solid-green curve.

tors act as an optical attenuator, which serves as a gain compensator for imbalanced outputs of the two detectors. The signals from high-speed photodetectors HPD1 and HPD2 were summed with a power combiner to produce DFI output signal, which was then monitored with a network analyzer (Anritsu MS4630B).

The DFI features of bidirectional MZIs were demonstrated with a transfer function measurement from noise/ signal simulators to DFI output. A swept-sine noise signal was provided by the internal oscillator of the network analyzer and was applied to the $\operatorname{EOM(s).~}$

The results for displacement-noise suppression are shown in Fig. 3. The transfer function of both detectors include the response functions of the simulating EOM, the photodetectors and optical and electronic phase delays from EOM through to the power combiner. Both amplitude and phase for the two signals were tuned to match each other using optical attenuators and path length adjustments so that the displacement-noise signal disappears in the DFI signal. About $30 \mathrm{~dB}$ of suppression of displacement noise was attained in a wide frequency region, while the maximum attained suppression was $45 \mathrm{~dB}$ at a particular frequency band, achieved when we tuned for maximum suppression in that region. Any imbalance between the two MZI signals determines the suppression ratio; it is believed that the subtly different frequency response of our two photodetectors, which cannot be compensated by simple optical attenuators or path length tuning, was the limiting factor here.

On the other hand, for the simulated-GW signal, the measured response function is shown in Fig. 4. Single detector outputs show response function in amplitude simi-

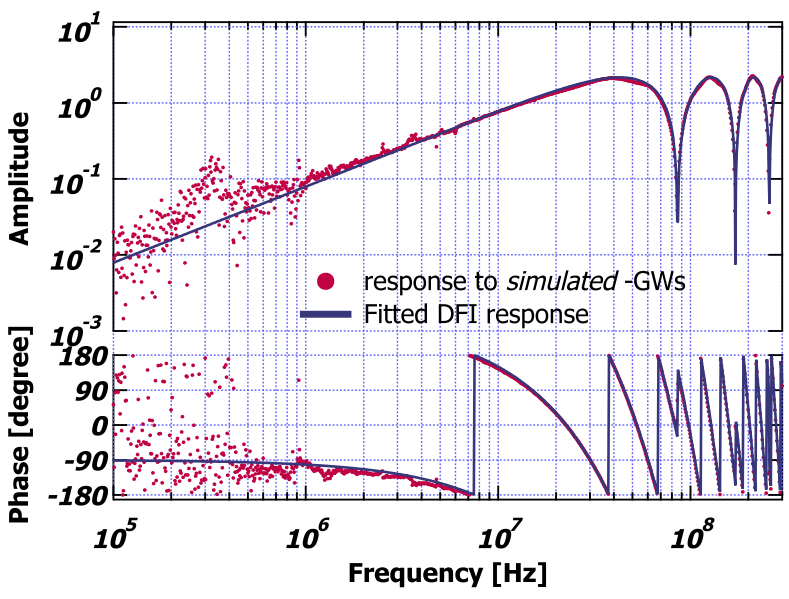

FIG. 5 (color online). The effects of the response functions of EOMs and HPDs on the DFI signal (solid-green curve in Fig. 4) were compensated by using the response function to single detector, shown in red dots. This corresponds to an intrinsic DFI (bidirectional MZIs for this experiment) response function to a simulated $\mathrm{GW}$ signal. The solid curve is a fitted DFI response giving reasonable fitting parameters.

lar to that for displacement noise; however, phase responses are different, which is what enables GW signal detection with DFI. As the DFI signal still contains the response functions of the simulating EOM and photodetectors, the measured transfer function was compensated with single detector data to remove them, then fitted with the theoretical function Eq. (7) as shown in Fig. 5. Fitted parameters, scale factor $A=2.16$ ( 2 in theory) and $l_{1 \alpha}-$ $l_{1 \beta}=3.50$ (3.55 in scale-measurement), agreed well with predicted values.

We thank Albrecht Rüdiger and Koji Arai for helpful discussions and comments. This research was partially supported by the Ministry of Education, Culture, Sports, Science and Technology under Grant-in-Aid for Scientific Research (B), No. 18340070, 2006. R. L. W. was supported by the U.S. National Science Foundation under Cooperative Agreement No. PHY-0107417. The research of Y.C., A.P., and K.S. is supported by the Alexander von Humboldt Foundation's Sofja Kovalevskaja Programme (funded by the German Ministry of Education and Research).

*Electronic address: sato.shuichi@nao.ac.jp

[1] M. Tinto, F. B. Estabrook, and J. W. Armstrong, Phys. Rev. D 65, 082003 (2002).

[2] S. Kawamura and Y. Chen, Phys. Rev. Lett. 93, 211103 (2004).

[3] Y. Chen and S. Kawamura, Phys. Rev. Lett. 96, 231102 (2006).

[4] Y. Chen et al., Phys. Rev. Lett. 97, 151103 (2006). 